\title{
Critique de la raison criminologique
}

Didier Bigo et Laurent Bonelli

\section{(2) OpenEdition}

Journals

Édition électronique

URL : http://journals.openedition.org/conflits/18879

DOI : 10.4000/conflits. 18879

ISSN : $1777-5345$

Éditeur :

CCLS - Centre d'études sur les conflits lilberté et sécurité, L'Harmattan

Édition imprimée

Date de publication : 31 décembre 2014

Pagination : 7-26

ISBN : 978-2-343-05760-6

ISSN : 1157-996X

Référence électronique

Didier Bigo et Laurent Bonelli, «Critique de la raison criminologique», Cultures \& Conflits [En ligne], 94-95-96 | été-automne-hiver 2014, mis en ligne le 20 février 2016, consulté le 30 mars 2021. URL : http://journals.openedition.org/conflits/18879; DOI : https://doi.org/10.4000/conflits. 18879 


\section{Critique de la raison criminologique}

\section{Didier BIGO, Laurent BONELLI}

Didier Bigo et Laurent Bonelli sont co-rédacteurs en chef de Cultures \& Conflits.

$\mathrm{P}$ our le sociologue ou le politiste français, l'expression même de « criminologie » sonne au premier abord comme un peu étrange et un peu ridicule. Pourquoi séparer des comportements - criminels en l'occurrence - de l'ensemble des relations sociales dans lesquelles ils sont encastrés et en faire un champ d'étude spécifique ? Si la criminologie se focalise seulement sur le crime, elle n'a guère plus de sens qu'une " anorexicologie », une « suicidologie » ou une "mariagologie », respectivement entendues comme sciences de l'anorexie, du suicide et du mariage. Si, en revanche, son ambition est de resituer le crime dans la fabrication et le maintien d'un ordre social et politique, pourquoi la distinguer de la sociologie ou de la science politique ? La question est volontairement naïve. Selon les pays, l'existence ou non de la criminologie comme discipline, de même que le sens qui y est donné dépendent de trajectoires historiques singulières dont il faut à chaque fois retracer les étapes et les modalités. Les rapports de forces sociaux et les équilibres académiques entre le droit, la sociologie, la médecine et la psychologie dessinent en effet des formes d'institutionnalisation différentes au Canada, en Belgique, aux ÉtatsUnis, au Royaume-Uni, en Allemagne, en Italie ou en France ${ }^{1}$. Toutefois, la naïveté de la question n'est qu'apparente. Les batailles pour l'existence disciplinaire de la criminologie demeurent en effet inséparables d'une promotion plus large de modes de questionnement, de catégories de pensée et de mise en récit spécifiques, c'est-à-dire d'une « raison criminologique » que ce numéro spécial de Cultures $\mathcal{E}$ Conflits entend interroger.

\section{Le crime, relation ou essence ?}

Au fond, faut-il ajouter quelque chose à l'analyse d'Émile Durkheim, lorsqu'il écrit en 1895 : «Classer le crime parmi les phénomènes de sociologie normale, ce n'est pas seulement dire qu'il est un phénomène inévitable quoi que regrettable, dû à l'incorrigible méchanceté des hommes ; c'est affirmer

1. Pour un propos général, voir Pires A., «La criminologie et ses objets paradoxaux : réflexions épistémologiques sur un nouveau paradigme », Déviance et société, 17 (2), 1993, pp. 129-161. 
qu'il est un facteur de la santé publique, une partie intégrante de toute société saine 2 » ? Pour le sociologue, la «normalité » du crime et l'étonnante plasticité des faits ou des individus englobés sous ce registre indique qu'il n'y a pas de neutralité et encore moins de naturalité de ces actes ou de ces identités. Ils résultent d'un processus de définition, dont l'enjeu est notamment de fixer les frontières morales d'un groupe ou d'une société. Cette perspective connaît une large postérité et inspire à des degrés divers les travaux d'auteurs aussi différents que Aaron V. Cicourel, Norbert Elias, Jean-Claude Chamboredon ou Donald Black, pour n'en citer que quelques uns ${ }^{3}$. Elle marque également ceux de Howard S. Becker et de Michel Foucault ${ }^{4}$, dont des contributions inédites ou peu connues sont présentées dans ce numéro.

Pour autant, cette approche n'a jamais complètement emporté l'adhésion de ceux qui ont partie liée à la régulation concrète de la criminalité et qui se reconnaissent plutôt dans Gabriel Tarde - souvent présenté comme l'un des pères de la criminologie (et lui même magistrat) - lorsqu'il affirme : « Le crime est un fait social comme un autre, mais un phénomène antisocial en même temps, comme le cancer participe à la vie d'un organisme, mais en travaillant à sa mort 5 ». La métaphore médicale n'apparaît pas ici par hasard : la raison criminologique, quelles que soient ses déclinaisons, demeure avant tout prophylactique. Aujourd'hui comme hier, il s'agit de fournir des savoirs pragmatiques, orientés vers l'action afin de "guérir » les sociétés de la criminalité. L'analyse glisse de la dimension relationnelle de définition du crime vers un caractère prétendument ontologique, c'est-à-dire vers sa substance, voire son essence. On attend alors dans cette vision du grand corps social malade, des réponses à des questions simples comme : «qui sont les délinquants ? » ou " quelles sont les causes de la criminalité ? " afin d' « apporter des solutions », toutes aussi simples, pour protéger la société.

\section{(In)fortunes de la criminologie française}

En France, la formation de savoirs sur le crime a été abondamment documentée et l'on ne peut en livrer qu'une synthèse imparfaite, qui demeure toutefois nécessaire pour fournir des éléments de compréhension des dynamiques contemporaines ${ }^{6}$. La constitution d'une discipline nommée anthropologie criminelle regroupant anthropologues, médecins, juristes et praticiens du pénal s'est d'abord focalisée dans les années 1880 autour de la controverse

2. Durkheim E., Les règles de la méthode sociologique, Paris, PUF 1996 [1895], p. 66.

3. Cicourel A. V., The Social Organization of Juvenile Justice, New Brunswick, Transaction Publishers, 1995 [1968] ; Elias N. et Scotson J. L., Les logiques de l'exclusion, Paris, Fayard, 1997 [1965] ; Chamboredon J-C., "La délinquance juvénile, essai de construction d'objet ", Revue française de sociologie, 12 (3), 1971, pp. 335-377 ; Black D., The Behavior of Law, San Diego, Academic Press, 1980 [1976].

4. Notamment Becker H. S., Outsiders. Études de sociologie de la déviance, Paris, Métailié, 1985 et Foucault M., Surveiller et punir. Naissance de la prison, Paris, Gallimard, 1975.

5. Tarde G., La philosophie pénale, Paris, éditions Cujas, 1972 (1890), p. 420.

6. Pour ces développements, nous renvoyons notamment à Kaluszynski M., La République à 
entre l'école italienne de Cesare Lombroso - qui évoque l'idée d'un criminelné - et celle d'Alexandre Lacassagne, qui lui reproche de ne pas tenir compte $\mathrm{du}$ « milieu social ». Mais la distance qui les sépare demeure minimale et c'est plutôt du côté de Gaston Richard, un collaborateur de Durkheim, que l'on trouvera les premiers travaux s'appuyant sur la statistique et l'objectivation du crime comme phénomène social 7 . Ceci dit, le projet ne connaîtra pas le succès que les durkheimiens eurent dans d'autres secteurs de l'activité scientifique. La rupture de Richard avec Durkheim en 1907, ainsi que la mort de Tarde sans héritiers en 1904, sonnent la quasi-disparition de recherches sociologiques en ce domaine, dans un contexte de montée en puissance du discours médical et psychiatrique, au sein même de l'anthropologie criminelle (avec notamment l'idée de la « dégénérescence»).

Le débat savant sur le crime oppose surtout à cette période juristes et médecins. Les premiers avaient reçu avec hostilité les thèses sur les doctrines biologiques de la criminalité, qui remettaient notamment en question des bases du droit pénal, que sont le libre-arbitre et la responsabilité. Ils voient également d'un mauvais oil l'irruption de l'expertise médicale dans les procès, capable par exemple de détruire l'expertise juridique basée sur un témoignage. L'anthropologie criminelle, vue comme discours savant (et maintenant médical) sur le crime est donc traitée avec suspicion. Derrière ces concurrences professionnelles se joue une lutte entre élites sociales (les médecins et les juristes) sur leur rôle et leur prédominance dans la société. L'une des manifestations de cette lutte est leur opposition sur les modalités disciplinaires à déployer pour juguler le crime : force du droit contre hygiène sociale et morale. La position dominante des juristes aux débuts de la Troisième République explique sans doute que leurs visions du monde l'aient emporté. $\mathrm{La}$ « criminologie » (nommée parfois « science criminelle» ou « science pénitentiaire ») connaît certes un début d'institutionnalisation, avec l'enseignement de la médecine légale, de la médecine mentale, d'éléments de police technique, d'anthropologie du droit pénal, mais dans les facultés de droit. Entre 1905 et 1914, trois d'entre-elles (Paris, Toulouse et Dijon) créent des certificats de science pénale et, en 1922, naît l'institut de criminologie de Paris, sous la double tutelle des facultés de droit et de médecine (mais dans les locaux de la faculté de droit).

l'éprenve du crime. La construction du crime comme objet politique, 1880-1920, Paris, L.G.D.J. 2002 ; Mucchielli L. (ed), Histoire de la criminologie française, Paris, L'Harmattan 1995, « L'impossible constitution d'une discipline criminologique en France. Cadres institutionnels, enjeux normatifs et développements de la recherche des années 1880 à nos jours ", Criminologie, 37 (1), 2004, pp. 13-42, Criminologie et lobby sécuritaire. Une controverse française, Paris, La Dispute, 2014 ; et Renneville M., Crime et folie. Deux siècles d'enquêtes médicales et judiciaires, Paris, Fayard, 2003.

7. Par exemple Richard G., «Les crises sociales et les conditions de la criminalité », L'Année sociologique, 1898-1899, année 3, pp. 15-42. 
La criminologie demeure ainsi académiquement marginale et marquée par les parcours conservateurs de ceux qui s'en réclament, comme par une profonde hostilité à un droit pénal fondé sur l'humanisme de la peine. Elle s'aliène donc nombre de juristes et se tourne vers la branche encore plus ténue de la science pénitentiaire. Si les thèses du criminel-né s'estompent, le lien entre classe sociale, pauvreté et criminalité fait en revanche florès. L'acte criminel supposerait une subjectivité criminelle particulière et la figure de l'ouvrier ou plus encore du lumpenprolétariat délinquant - envahit les discours. Les questionnements se déportent vers les politiques à adopter lorsqu'existent des situations criminogènes qui favorisent l'émergence de types d'individus associés à des actes délinquants particuliers. Deux approches s'opposent ou se combinent pour combattre ce que l'on appelle alors « la dangerosité 8 » : d'une part la « prophylaxie sociale 9 » qui entend jouer sur les conditions individuelles et collectives pouvant faciliter la délinquance et, de l'autre, les mesures de sûreté visant à « réadapter " l'individu déviant en plus de son châtiment pénal. Ces dimensions structurent les disputes criminologiques après la Seconde Guerre mondiale, auxquelles on peut associer les noms de Jean Pinatel (1913-1999), inspecteur de l'administration pénitentiaire, collaborateur assidu de la Revue de science criminelle et de droit pénal comparé et membre important de la Société internationale de criminologie ; du magistrat Marc Ancel (1902-1990), fondateur de « la défense sociale nouvelle 10 » ou encore de Jacques Léauté, professeur de droit privé à la Faculté de droit de Paris (19581970), puis directeur de l'Institut de criminologie de Paris (1972-1985), qui insiste sur le rôle de la criminologie clinique, sur les déterminants individuels du crime, et sur la nécessité de lier l'approche psychologique et le traitement judiciaire ${ }^{11}$. Malgré cette activité - y compris internationale -, c'est pourtant du côté de la sociologie et du droit que se développent en France les études sur le crime, d'abord avec Henri Lévy-Bruhl (1884-1964), puis avec André Davidovitch et Jean Carbonnier ${ }^{12}$. Ce mouvement s'amplifie à partir des années 1960-1970, dans un contexte intellectuel marqué par la contestation politique du système pénal et la réception des théories interactionnistes et notamment de celle de l'étiquetage ${ }^{13}$. La dynamique se déporte davantage vers l'étude de la pénalité, d'abord au Service d'études pénales et criminologiques (SEPC), créé en 1968 sous la houlette de Philippe Robert, puis au

8. Danet J., «La dangerosité, une notion criminologique, séculaire et mutante », Champ pénal/Penal field [en ligne], vol. V, 2008.

9. Le premier congrès international de prophylaxie criminelle se tient à Paris du 27 au 30 septembre 1959. Il entend «substituer une science tout à fait nouvelle aux anciennes conceptions en matière de prévention du crime et des psychopathies ". Cf. Revue internationale de droit comparé, 11 (2), 1959. p. 422.

10. Ancel M., La Défense sociale nouvelle, un mouvement de politique criminelle humaniste, Paris, éditions Cujas, 1954.

11. Léauté J., Criminologie et science pénitentiaire, Paris, PUF, 1972.

12. Mucchielli L. et Marcel J-C., «La sociologie du crime en France depuis 1945 », in Mucchielli L. et Robert Ph., Crime et sécurité. L'état des savoirs, Paris, La Découverte, 2002.

13. Voir Robert Ph. « La sociologie entre une criminologie du passage à l'acte et une criminologie de la réaction sociale ", L’Année sociologique, troisième série, vol. XXIV, pp. 441-504. 
Centre de recherche sociologique sur le droit et les institutions pénales (CESDIP), qui le remplace en 1983. La criminologie prophylactique continue certes à irriguer des milieux professionnels, mais malgré les désirs de Pinatel ${ }^{14}$, ne parvient guère à se ménager des espaces autonomes, au-delà de quelques îlots disséminés dans les facultés de droit.

Vers la fin des années 1970, l'Institut de criminologie de Paris emprunte une voie originale pour sortir de son confinement. Léauté essaie d'y préserver l'essence de la question " criminelle », mais en la rattachant au discours naissant sur le «terrorisme », qui accompagne le développement de la violence politique en Europe (en Allemagne, en Italie, mais aussi liée à la question palestinienne). L'ethnologue Jean Servier, partisan à son heure de l'Algérie française, s'y associe occasionnellement ${ }^{15}$. Surtout, le journaliste Christian de Bongain - un ancien d'Occident chrétien, plus connu sous son pseudonyme Xavier Raufer - y est recruté pour mener des recherches.

Ce dernier joue très vite le rôle d'intermédiaire entre l'Institut, des membres des services de renseignements français avec lesquels il partage des affinités politiques et certains médias. Dans des articles de presse (à L'Express notamment), des émissions de radio et de télévision et dans des ouvrages (plus d'une vingtaine entre 1982 et 2010), il développe ses thèses sur le terrorisme et le crime sous toutes leurs facettes. Utilisant un langage simplificateur, dont il s'honore dans une veine anti-intellectuelle, il fait un usage abondant des métaphores biologiques et médicales ${ }^{16}$. Sous sa plume, les organisations clandestines deviennent des « guérillas dégénérées 17 », les États du Sud, des «États faillis » aux «zones grises incontrôlables » justifiant les interventions des puissances occidentales, et il insiste sur les ennemis infiltrés dans ces dernières, hier les « Partis communistes combattants », aujourd'hui les « islamo-gangsters ». La «violence sociale » et les «zones de non droit» des banlieues françaises sont projetées dans des discussions géopolitiques sur le chaos global, dessinant l'image d'un présent et d'un futur menaçants. Sa prose et ses gloses mêlent la désignation de " groupes à risques ", l'évaluation des conditions de leur passage à l'acte et des injonctions à l'action (publique). Il suit en cela la ligne de pente de la criminologie prophylactique de ses ainés, sans toutefois se

14. Pinatel J., « La criminologie : recherche scientifique et action sociale », Revue française de sociologie, 1964, 5-3, pp. 325-330.

15. En 1979, il écrit notamment un «Que sais-je ? " intitulé Le terrorisme ( ${ }^{\circ} 1768$ ) dans lequel on peut lire que «La personnalité du terroriste est comme celle de certains criminels de droit commun : une personnalité suicidaire. Ce refus du présent, de la société des adultes, cette volonté de retour au passé sont en réalité autant de jalons sur un même itinéraire de fuite vers la mort, solution de tous les échecs » (p. 121 de l'édition de 1992).

16. Sur les métaphores médicales, l'appartenance à l'extrême droite et l'anthropologie guerrière, voir Cultures E Conflits n43, 2001, intitulé «Construire l'ennemi intérieur ».

17. Métaphores associées aux théories des conflits de basse intensité développées par David Galula et Max Manwaring dont il s’inspire sans toutefois leur reconnaître la paternité de ces « notions ». Voir notamment, Galula D., Counterinsurgency Warfare: Theory And Practice, New York, Praeger, 1964 et Manwaring, M. G., Gray Area Phenomena: Confronting the New World Disorder, Boulder, Westview press 1993. 
référer à eux ni prendre part aux débats intellectuels qui les mobilisent. Dans des médias friands d'interprétations simples et «à chaud », ce type de propos génère une notoriété immédiate. Et ce n'est pas le moindre des paradoxes que le renouvellement de la criminologie française auquel il participe repose davantage sur des ressources médiatiques et politiques que sur celles académiques et professionnelles qui prévalaient auparavant.

\section{La « criminologie nouvelle » à l'offensive}

À partir de la fin des années 1980, dans une configuration qui voit la consolidation d'une préoccupation publique pour la sécurité ${ }^{18}$, une remise en cause des analyses stratégiques due à la fin de la bipolarité, ainsi que le resserrement des liens d'interdépendance entre les champs médiatique et politique ${ }^{19}$, ces formes d'expertise criminologique vont prospérer.

Assénant des affirmations idéologiques comme s'il s'agissait d'évidences venant d'un savoir académique, ceux qui peuvent les endosser arrivent à créer un genre journalistique et télévisuel qui fait leur succès : une démagogie disciplinaire d'un genre nouveau où l'expert présumé - loin de se distancier des stéréotypes - flatte au contraire un «bon sens » dit populaire dès que ce dernier peut être orienté vers le soutien aux politiques les plus sécuritaires. Utilisant un style narratif facile et racoleur, mêlant fiction et détails réels, où les ennemis sont presque toujours secrets, puissants et sur le point de renverser un système inconscient du danger («l'aveuglement » et «l'angélisme » reviennent souvent ${ }^{20}$ ), ils participent à la promotion de ce que le politiste américain Michael Rogin nomme une «démonologie politique 21 ». Celle-ci rallie d'anciens professionnels de la sécurité qui peuvent pimenter à moindre coût la publication de leurs mémoires et des hommes politiques soucieux de marquer des écarts distinctifs dans leurs partis en endossant la cause de la « sécurité ».

Ces nouveaux experts déploient ainsi leurs « analyses » sur les plateaux de télévision, dans les studios de radios ou les colonnes des journaux; dans le champ politique (comme conseillers des élus locaux, de partis politiques ou des cabinets ministériels); dans le champ bureaucratique (par la participation aux écoles de formation de la police ou de la gendarmerie, à des missions techniques, des rapports) ; dans le champ éditorial (par des publications d'ouvrages, la direction de collections) et à la marge du champ académique (par des enseignements universitaires - notamment dans des masters 2 professionnels ou des diplômes universitaires (DU) spécialisés en sécurité) ${ }^{22}$. Selon une

18. Bonelli L., La France a peur. Une histoire sociale de "l'insécurité », Paris, La Découverte 2010 [2008].

19. Champagne P., Faire l'opinion. Le nouveau jeu politique, Paris, éditions de Minuit, 1990.

20. Par exemple Raufer X., « Temps, espace : horizon stratégique », Sécurité globale, 5, 2013, p. 15.

21. Rogin M., Les démons de l'Amérique, Paris, Seuil, 1998.

22. Bonelli L., "Quand les consultants se saisissent de la sécurité urbaine », Savoir / Agir, 9, 
logique circulaire, chaque position occupée dans l'un de ces univers renforce leur autorité à intervenir dans les autres, tout en contribuant à y diffuser l'image d'une criminologie "en prise avec son temps ", accessible dans ses analyses et parfois iconoclaste dans son propos. La création par le gouvernement d'une chaire de "criminologie appliquée » au Conservatoire national des arts et métiers (CNAM) en 2009 puis d'une section de « criminologie » (section 75) au Conseil national des universités (CNU) en 2012 viennent objectiver l'augmentation de la valeur d'usage et de la valeur d'échange de cette criminologie qui ignore son histoire. Si quelques universitaires (notamment des héritiers de Pinatel) s'associent un temps à l'entreprise, le label est surtout approprié par quelques entrepreneurs de cause dont les enjeux demeurent plus moraux, économiques et politiques qu'académiques. Ce dont atteste d'ailleurs leur absence d'adhésion, même minimale, aux règles de fonctionnement du champ scientifique (d'illusio et de libido pour reprendre les termes de Pierre Bourdieu 23 ), leur coupure avec les cercles antérieurs où se débattaient ces questions (comme par exemple l'Association française de criminologie) et leur ignorance tenace des travaux criminologiques antérieurs.

La trajectoire de M. Alain Bauer, longtemps président directeur général d'une entreprise de conseil en sécurité ( $\mathrm{AB}$ Associates) et aujourd'hui titulaire de la chaire du CNAM - il aime à ce titre se présenter comme «le seul professeur de criminologie français » - condense à ce point l'ensemble de ce mouvement qu'il en vient presque à le personnifier. Son style « bonhomme » et son sens de la formule lui permettent de ravir des publics divers, en particulier les médias, les entrepreneurs de sécurité, les élus locaux et le grand public ${ }^{24}$. Mais l'autorité qu'il a acquise n'est pas séparable du travail collectif d'un petit groupe pour asseoir ses positions et qui fonctionne par des transferts croisés de capital social et symbolique (incluant des renvois d'ascenseur).

Parmi ses membres, M. Bauer ne manque jamais de citer M. Raufer (qui se fera conférer finalement un titre de docteur en géopolitique en 2007), M. François Haut, maître de conférences en droit, et M. Yves Roucaute, professeur de sciences politiques à l'université de Paris Ouest Nanterre ${ }^{25}$. Les deux premiers s'occupent du département de recherche sur les menaces criminelles contemporaines (DRMCC) à l'Institut de criminologie de Paris, qui a

septembre 2009, pp. 17-28.

23. Bourdieu P., Méditations pascaliennes, Paris, Seuil, 2003 [1997], p. 25.

24. On notera en revanche le dédain des universitaires venant de la criminologie universitaire critique ou non - qui s'est développée en Angleterre, en Italie, en Suède lorsqu'il représente la criminologie française dans des enceintes spécialisées comme Europol, et les distances que les responsables non français de ces différentes agences maintiennent avec lui et avec ses " manières ». Sur sa trajectoire, voir Rimbert P., « Les managers de l'insécurité. Production et circulation d'un discours sécuritaire ", in Bonelli L. et Sainati G (ed), La machine à punir. Pratiques et discours sécuritaires, Paris, L’Esprit frappeur, 2001, pp. 235-276 et Bonelli L., La France a peur..., op. cit., pp. 169-172, 241.

25. Par exemple dans Bauer A., À la recherche de la criminologie : une enquête, Paris, CNRS éditions, 2010, pp. 27 et 33. 
succédé en 1997 au CERVIP (Centre d'étude et de recherche sur la violence politique, créé en 1986). Le DRMCC rattaché par contrat à l'université Panthéon Assas - Paris II est habilité à délivrer un diplôme d'université (DU). Les intervenants sont essentiellement des professionnels de la sécurité et son objet reste explicite : «la détection précoce, l'observation et l'analyse de toutes les formes de menaces criminelles aux fins de proposer des diagnostics, de dégager des concepts et de mesurer leur impact 26 ». Surtout, le DRMCC dispose d'un débouché éditorial important aux Presses universitaires de France (PUF), où M. Raufer avait été recruté au début des années 1990 et où il dirige la collection «Criminalité internationale ». La singularité de cet assemblage institutionnel (associatif / éditorial / universitaire) doit beaucoup aux affinités politiques nationalistes et anticommunistes de ceux qui le composent et l'ont rendu possible ${ }^{27}$. Mais il n'en reste pas moins qu'il offre à M. Bauer des débouchés qui lui étaient inaccessibles au début de sa carrière. Ainsi, c'est aux PUF qu'il publie en 1998 son premier ouvrage Violences et insécurités urbaines (avec M. Raufer) et qu'il peut ensuite s'associer avec des hauts responsables policiers, comme M. Émile Perez (avec qui il cosigne L'Amérique, la violence, le crime. Les réalités et les mythes en 2000, Les polices aux ÉtatsUnis et Le crime aux États-Unis en 2003, puis Les 100 mots de la police et du crime en 2009), M. André-Michel Ventre (Les polices en France. Sécurité publique et opérateurs privés en 2001) ou le juge Jean-Louis Bruguière (Les 100 mots du terrorisme, "Que sais-je ? ", $\left.\mathrm{n}^{\circ} 3897,2010\right)$. Il y publie ensuite 14 ouvrages entre 2010 et 2014, dont un fameux Criminologie plurielle (2011), que le criminologue belge Dan Kaminski résume comme une tentative d'établir une "science de la menace et de l'ennemi », avant de le congédier d'une belle formule : "une "science" de la menace est une menace pour la science ; une “science" de l'ennemi est une ennemie de la science 28 ».

Ce transfert de capital académique s'observe également dans son rapprochement avec M. Roucaute, un professeur agrégé de science politique de l'université de Paris Ouest Nanterre, où il dirige un master 2 de « management du risque ». Celui-ci signe en 2008 avec MM. Bauer et Raufer le court texte programmatique d'une «criminologie refondée », dont l'ambition est « de répondre aux questions fondamentales - qui sont aujourd'hui les criminels (et comment évoluent-ils) ? Où sont-ils ? Combien sont-ils ? Que font-ils et pourquoi ? 29 » en intégrant également des perspectives de géopolitique et de relations internationales.

26. http://www.drmcc.org

27. Rigouste M., Les marchands de peur. La bande à Baner et l'idéologie sécuritaire, Paris, Libertalia, 2011.

28. Kaminski D., "Criminologie plurielle et pourtant singulière », Revue de science criminelle et de droit pénal comparé, 2, 2011, pp. 475-495.

29. Bauer A., Raufer X. et Roucaute Y., «Une vocation nouvelle pour la criminologie », Sécurité globale, 5, 2008, p. 90. 
M. Bauer tire de ces rapprochements une légitimité intellectuelle par imprégnation ou par capillarité. Ils lui fournissent des ressources dans les luttes qu'il conduit dans les bureaucraties de la sécurité. En effet la «nouvelle criminologie » française - comme il l'appelle - apparaît peut-être moins comme une entreprise proprement universitaire qu'administrative et politique. Fort des proximités nouées avec des élites de ces secteurs, M. Bauer va jouer un rôle dans la réorganisation des institutions de production et de diffusion de connaissances sur la criminalité ${ }^{30}$.

D'abord, il participe à la réorientation de l'Institut des hautes études sur la sécurité intérieure (IHESI) du ministère de l'Intérieur, créé en 1989 par M. Pierre Joxe et rebaptisé Institut national des hautes études sur la sécurité (INHES) en 2004 : un Observatoire national de la délinquance (OND) y est créé, dont M. Bauer devient le président. Ce nouveau département composé de 9 personnes est dirigé par M. Christophe Soullez, un ancien étudiant de M. Raufer à l'Institut de criminologie de Paris. Il recrute également comme chargé d'études un ancien directeur de la sécurité de la ville de Roubaix, M. Jean-Luc Besson, auteur d'un ouvrage intitulé Les cartes du crime, publié la même année aux PUF, et préfacé par... M. Bauer. Comme le rapporte un témoin, cette petite cellule prit rapidement le pas sur le conseil d'orientation de l'OND et devint la véritable cheville ouvrière de la production, de l'analyse et de la communication des chiffres de la délinquance ${ }^{31}$. Le poids pris par les promoteurs de l'OND dans l'INHES se renforce encore lorsque ce dernier devient l'Observatoire national de la délinquance et des réponses pénales (ONDRP) en 2009, à l'occasion d'un nouveau changement de nom de l'institut, désormais appelé Institut national des hautes études sur la sécurité et la justice (INHESJ) et dirigé jusqu'en mars 2014 par l'inspecteur général de la police nationale André-Michel Ventre, coauteur de M. Bauer aux PUF.

Cette influence se traduit également sur le terrain des connaissances. À son origine, l'Institut laissait généralement une large place à la recherche, même si les relations entre administration et chercheurs étaient parfois rugueuses ${ }^{32}$. À partir de 2004, le département recherche perd son autonomie, se voit adjoindre les études et est dirigé par des professionnels : commissaire de police puis magistrat. La revue qu'il éditait (Les cabiers de la sécurité intérieure), d'une bonne tenue académique, est réorganisée et devient en septembre 2007 Les cabiers de la sécurité. M. Roucaute en devient, pour un temps, le

30. M. Bauer est enchâssé dans une multitude de réseaux politiques (au Parti socialiste comme à l'Union pour un mouvement populaire) et francs-maçons (il fut grand maître du Grand Orient de France de 2000 à 2003) qui participent à accroître sa surface sociale.

31. Ocqueteau F., «Une machine à retraiter les outils de mesure du crime et de l'insécurité : l'Observatoire National de la Délinquance », Droit E Société, LGDJ, 2012, pp. 447-471.

32. Ocqueteau F. et Monjardet D., "Insupportable et indispensable, la recherche au ministère de l'Intérieur ", in Bezes Ph., Chauvière M., Chevallier J., de Montricher N. et Ocqueteau F., L'État à l'éprenve des sciences sociales. La fonction recherche dans les administrations sous la ve République, Paris, La Découverte, 2005, pp. 230-247. 
rédacteur en chef et MM. Raufer et Haut figurent parmi les contributeurs très réguliers. L'inflexion des problématiques est sensible et laisse une large place au projet intellectuel de la nouvelle criminologie. La simple liste des titres est évocatrice : « La violence des mineurs $\gg\left(\mathrm{n}^{\circ} 1\right.$, septembre 2007); «Le fléau de la drogue » $\left(n^{\circ} 5\right.$, septembre 2007); « La criminalité numérique $"\left(\mathrm{n}^{\circ} 6\right.$, décembre 2008) ; "Sport : menaces et risques 》 $\left(\mathrm{n}^{\circ} 11\right.$, mars 2010) ; «Les dangers de la contrefaçon» (n¹5, mars 2011); "L'école face aux défis de la sécurité » (n`16, juin 2011) ; « L'économie du crime $\gg\left(n^{\circ} 25\right.$, septembre 2013$)$; etc. Parfois, la couverture en dit assez long sur le contenu, comme le montre celle du $\mathrm{n}^{\circ} 7$, intitulé «Les organisations criminelles » (mars 2009), reproduite cicontre.

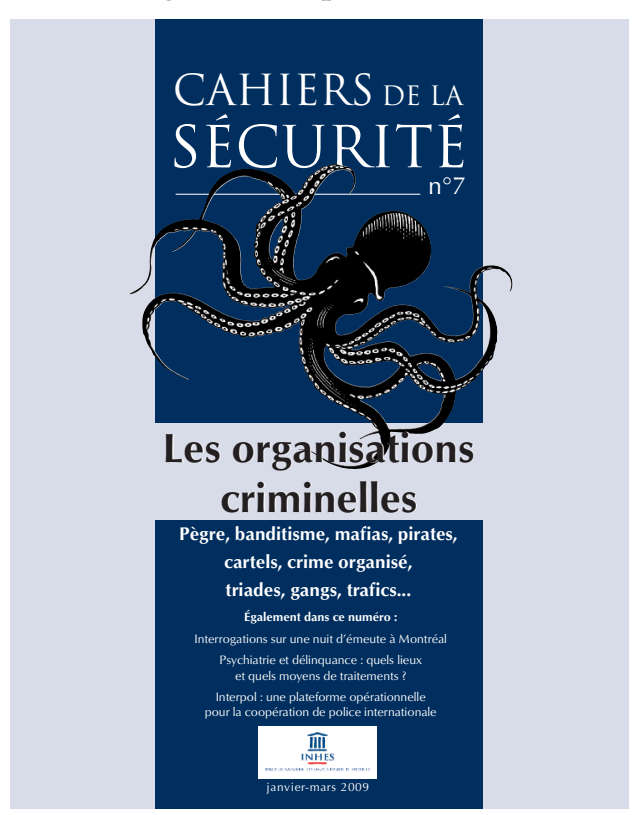

La stratégie de conquête des promoteurs de la «nouvelle criminologie » ne s'arrête pas aux institutions du ministère de l'Intérieur. En 2007, M. Bauer se voit confier par M. Nicolas Sarkozy et M. François Fillon la présidence d'une mission de réflexion sur le rapprochement des institutions de formation et de recherche qui travaillent sur les questions de sécurité et de défense. Parmi ses cinq membres, on remarque M. Raufer, et l'un des deux rapporteurs n'est autre que M. Soullez. La mission rend son rapport en mars 2008, préconisant la fusion de l'INHES et de l'Institut des études et recherches sur la sécurité des entreprises (IERSE) d'une part et celle du Centre des hautes études de l'armement (CHEAr) avec l'Institut des hautes études de la défense nationale (IHEDN) d'autre part. Il propose également la création d'un Conseil supérieur de la formation et de la recherche stratégique (CSFRS), en charge de fixer les orientations stratégiques des deux pôles et de coordonner leur activité, notamment en regroupant l'ensemble de ces institutions sur un même site, l'École militaire. L'ensemble du projet est mis en œuvre entre 2009 et 2010, et le président du CSFRS est... M. Bauer. M. Raufer n'est bien sûr pas oublié. Il reçoit une place de choix, comme secrétaire du " groupe des experts internationaux », en même temps qu'une tribune annuelle, lors des Assises nationales de la recherche stratégique. Outre le prestige associé à ces positions, les enjeux qui y sont attachés sont également extrêmement pratiques, puisque le CSFRS est devenu un guichet unique par lequel passent nombre de financements de recherche auparavant distribués par les institutions qui le composent. Une situation avantageuse que laisse transparaître M. Bauer, lorsqu'il déclare lors 
des Assises du 24 juin 2010 : «Le CSFRS n’a pas vocation à monopoliser ou uniformiser la recherche, mais à lui permettre de se développer dans les meilleures conditions en évitant redondances et gaspillage 33 ».

La colonisation rapide d'un bon nombre d'administrations produisant des savoirs sur la sécurité par les tenants d'une nouvelle criminologie ne signifie pas que les relations en son sein soient exemptes de tensions, ou qu'elle rassemble seulement des acteurs tendus vers un même objectif. Comme toute mobilisation collective, cette entreprise agrège des intérêts hétérogènes et fait l'objet d'investissements inégaux. Elle se heurte de surcroît à des résistances. Les agents du ministère de la Justice semblent n'accompagner le mouvement que du bout des lèvres et maintiennent de larges marges d'autonomie, allant par exemple jusqu'à créer en 2014 un Observatoire de la récidive et de la désistance en dehors de l'ONDRP. De la même manière, les institutions militaires semblent avoir assez largement freiné ce que certains en leur sein considèrent comme une offensive du ministère de l'Intérieur.

Il n'en reste pas moins qu'entre 2004 (création de l'OND) et 2009 (création du CSFRS et de la chaire du CNAM), les positions institutionnelles des partisans d'une «criminologie renouvelée » ont été largement renforcées. Et c'est dans ce contexte que Mme Valérie Pécresse, ministre de l'enseignement supérieur et de la recherche confie en décembre 2009 à M. Loïck Villerbu, un professeur de psychologie à Rennes-II, la présidence d'une «Conférence nationale de criminologie ». Parmi les neuf membres du comité, on remarque l'incontournable M. Bauer. Le rapport, rendu en juin 2010, préconise le développement de la criminologie dans les universités françaises. Une section nouvelle du CNU (la 75) voit le jour par décret le 13 février 2012, dont les membres sont nommés par le ministère le 18 avril. MM. Bauer et Haut font évidemment partie des 18 titulaires. La conjonction des mobilisations et de l'alternance politique emporte la section, supprimée en août sans avoir eu d'activité ${ }^{34}$. La sociologie de cette querelle - qui coalise des groupes assez différents - reste à faire. Mais l'intention politique qui lui a donné naissance était claire : renforcer et stabiliser les positions académiques des tenants de la «nouvelle criminologie ».

L'échec de la section 75 ne signe pour autant pas celui de cette dernière dont l'objet demeure moins scolastique que symbolique. Il s'agit en effet moins de débats intellectuels que de la constitution d'une identité académique en simili, capable de faire illusion vis-à-vis des professionnels de la sécurité, de la politique et des médias ${ }^{35}$. En se parant des vertus de neutralité et de désin-

33. Cité dans Moro Ch., «Premières Assises nationales de la recherche stratégique : bilan et perspectives ", Sécurité globale, 13, 2010, p. 18.

34. Mucchielli L., Criminologie et lobby sécuritaire..., op. cit., pp. 149 et ss.

35. La dernière manifestation en date de ces tentatives qui « font science » est le lancement en octobre 2013 de la Revue française de criminologie et de droit pénal (RFCDP), dont le titre et l'apparence austère rappellent les revues académiques. En réalité, elle dépend de l'Institut 
téressement attachées à la science, celle-ci peut en effet atténuer les suspicions qui pèsent sur le caractère idéologique et/ou commercial des analyses des " nouveaux criminologues »et, par là même, renforcer les positions qu'ils occupent dans différents champs (bureaucratique, médiatique et politique notamment). D’ailleurs, on ne peut qu'observer avec surprise la cécité de nombre de chercheurs mobilisés contre la section 75, qui tenaient pour entité négligeable les menées de M. Bauer et ses alliés tant qu'elles avaient lieu en dehors du champ académique et les découvrent horrifiés quand elles y entrent par effraction du fait du prince. Leur indignation prête d'abord le flanc à la critique facile de la défense d'un territoire, mais surtout rate l'essentiel : l'analyse des rationalités d'une raison criminologique prégnante bien au-delà de la discipline éponyme, dans l'administration, les médias, la politique et même parfois dans la science politique (particulièrement dans l'étude des politiques publiques), la sociologie, le droit ou la psychologie.

\section{(Dé)raison criminologique contemporaine ? Continuité, critique, aban- don}

Si l'on met momentanément de côté ces aléas récents et bien français, en adoptant une perspective de moyenne durée, on observe que la raison criminologique prophylactique s'est transformée et ajustée aux pratiques de management observables dans le cadre d'une bureaucratisation néolibérale ${ }^{36}$. Elle a d'abord rompu en partie avec les thèses de l'individu dangereux sur lesquelles Foucault portait un diagnostic critique, et elle n'est plus véritablement un carrefour central entre savoirs pénitentiaire et psychiatrique. Elle apparaît davantage en adéquation avec les savoirs managériaux, les entreprises privées de sécurité, les technologies de géolocalisation, de cartographie, de vidéo-surveillance, de logiciels de profilage. Elle devient également un discours de justification de la surveillance technologique et des demandes policières d'un accès sans réserve à des données (personnelles) numérisées. Elle maintient son soidisant utilitarisme (issu de la « défense sociale »), tout en se dissociant des éléments les plus extrémistes qui l'avaient associée avec l'idéologie fasciste et la biologisation des populations ${ }^{37}$. Il subsiste certes à ses marges une attraction pour le « criminel né », jadis repéré par la forme de son crâne et aujourd'hui par les neurosciences ${ }^{38}$. Mais l'orientation semble plus «comportementa-

pour la Justice, une association de type loi de 1901, connue pour ses analyses très conservatrices en matière de justice pénale. MM. Bauer et Raufer font partie des comités scientifique et de lecture aux côtés de quelques universitaires (souvent retraités, comme le criminologue canadien Maurice Cusson ou le professeur de droit public André Varinard) et surtout de praticiens.

36. Hibou B., La bureaucratisation du monde à l'ère néolibérale, Paris, La Découverte, 2012.

37. Sur les dilemmes de la criminologie à se départir de ces tendances en Allemagne, on se reportera à la contribution de Grégory Salle dans ce numéro.

38. Bien qu'elles s'en défendent souvent, un nombre important de publications vont en ce sens, dans des revues académiques qui n'ont rien de confidentielles. Voir par exemple Sterzer Ph., "Born to Be Criminal? What to Make of Early Biological Risk Factors for Criminal Behavior”, American Journal of Psychiatry, 167 (1), janvier 2010, pp. 1-3 ; Templer D. et 
liste ", afin de recruter certains médecins et psychiatres spécialisés dans la 《détection précoce» des comportements «à risques» 39 . Le comportementalisme devient moins une caractéristique intrinsèque à un individu particulier, qu'une relation distinguant au sein d'une population ceux qui ont des comportements « anormaux ».

Ceci permet de mobiliser des savoirs statistiques pour la gestion des populations et, comme le signale Ian Hacking, de la crédibiliser par des chiffres et des courbes, distribuant les individus entre points extrêmes et points modaux et constituant ainsi des frontières entre catégories. La répartition statistique des individus aux positions rares est relue comme une anormalité par rapport à des normes sociales majoritaires et partant, comme une illégitimité 40 . Ce saut logique, que Foucault avait désigné comme la différence entre normation et normalisation, est loin d'être l'apanage de la criminologie. On le retrouve dans la plupart des sciences de gestion, mais il prend une tournure particulière avec le mode de traitement que celle-ci recommande à l'égard de ces populations minoritaires, désormais perçues davantage comme migrants à surveiller et mettre à l'écart, plutôt que comme prolétaires à discipliner, les deux objectifs ayant connu une inversion de priorité, mais subsistant comme fondement anthropologique de cette raison criminologique et de son mode de production de la vérité ${ }^{41}$.

La conjonction entre la promotion d'une surveillance dissuasive, préventive et prédictive 42 et des processus contemporains d'(in)sécurisation dédifférenciant les sphères du policier, du renseignement, du militaire et de l'entre-

Rushton Ph., "IQ, skin color, crime, HIV/AIDS, and income in 50 U.S. states", Intelligence, 39, 2011, pp. 437-442 ; Yang Y. et alii, "Volume Reduction in Prefrontal Gray Matter in Unsuccessful Criminal Psychopath", Biological Psychiatry, 57, 2005, pp. 1103-1108; Yang Y. et alii, "Localization of Deformations Within the Amygdala in Individuals With Psychopathy", Archives of General Psychiatry, 66 (9), 2009, pp. 986-994 ; Meyer-Lindenberg A. et alii, "Neural mechanisms of genetic risk for impulsivity and violence in humans", Proceedings of the National Academy of Sciences, 103 (16), 2006, pp. 6269-6274. Un auteur important de ce courant est Adrian Raines, auteur notamment de The Anatomy of Violence: The Biological Roots of Crime, New York, Pantheon, 2013. Nous remercions Jean-Claude Croizet, de l'université de Poitiers, pour ses éclaircissements sur ce domaine.

39. On trouvera par exemple une magnifique « courbe évolutive d'un jeune qui au fur et à mesure des années s'écarte du "droit chemin" pour s'enfoncer dans la délinquance » dans Bénisti A., Sur la prévention de la délinquance, Rapport de la Commission prévention du groupe d'études parlementaire sur la sécurité intérieure de l'Assemblée nationale, octobre 2004, p. 7. Un rapport de l'INSERM, intitulé Trouble des conduites chez l'enfant et l'adolescent, de septembre 2005 allait dans le même sens. Il souleva une large opposition de pédiatres, de pédopsychiatres et de psychiatres. La pétition « Pas de zéro de conduite pour les enfants de trois ans! 》 qu'ils ont lancée a recueilli près de 200000 signatures.

40. Hacking I., "Statistical Language, Statistical Truth and Statistical Reason: The SelfAuthentication of a Style of Reasoning", in McMullin E. (ed), Social Dimensions of Science, Notre Dame, University of Notre Dame Press, 1992, pp. 130-157 et "'Language, Truth and Reason' thirty Years Later", Studies in History and Philosophy of Science, 43, 2012, pp. 599609.

41. Voir la contribution de Fabienne Brion dans ce numéro.

42. Bigo D., "Sécurité maximale et prévention ? La matrice du futur antérieur et ses grilles ", in Cassin B. (ed), Derrière les grilles : sortir du tout évaluation, Fayard, Paris, 2013. 
prise ${ }^{43}$ a permis à la « nouvelle criminologie » de se démarquer de ses origines contestées et d'élargir son assise. Elle apporte à un discours stratégique parlant d'ennemi interne un formidable alibi, puisqu'elle affaiblit les différenciations entre adversaires et ennemis, ennemis et criminels, populations dangereuses et populations en danger, situations risquées et situations nécessitant la résilience. Désormais, explique savamment $M$. Bauer après les attentats des 7 , 8 et 9 janvier 2015, " la menace provient de groupes hybrides et opportunistes capables de transformations rapides. Il existe un nouveau "melting-pot" criminel intégrant fanatisme religieux, massacres, piraterie, trafic d'êtres humains, de drogues, d'armes, de substances toxiques ou de diamants. Un continuum criminalo-terroriste, un "gangsterrorisme" apparaît, qui ne correspond plus aux petits casiers doctement préparés pour eux » (Le Point, 15 janvier 2015).

De manière plus centrale encore, la raison criminologique participe, de pair avec les rationalités pratiques des services de renseignement et de la surveillance à grande échelle, à invalider la logique du procès pénal ou à la subvertir, à s'appuyer sur les pratiques proactives de police et à s'adosser sur une production de «l'opinion » qui aurait peur, se sentirait troublée et inquiète. Il n'est donc pas surprenant qu'outre les sciences de gestion, elle s'appuie sur celles de la communication et renoue avec les théories des opérations psychologiques des guerres antisubversives. Il s'agit cette fois de frapper l'imagination en construisant la figure du criminel comme un ennemi de l'intérieur qui a néanmoins des caractéristiques d'altérité suffisamment reconnaissables (retrouvant par là les ressorts classiques de la propagande) pour que chacun puisse se convaincre du bien-fondé de ses analyses et de ses verdicts. Par exemple quand elle propose la mise à l'écart et l'expulsion de l'individu suspect comme alternative économique (et morale) à l'enfermement de longue durée du coupable, au nom du fait que la pénologie devrait dorénavant moins s'intéresser à la peine et la culpabilité qu'à la dangerosité sociale du phénomène criminel et à sa prévention ${ }^{44}$. Une prévention qui supposerait diverses mesures de sureté, dans et hors le droit, le futur ne s'encadrant pas juridiquement.

43. Voir notamment les n 58 de Cultures $\mathcal{E}$ Conflits (2005) sur «Suspicion et exception » et 3132 (1998) sur « Sécurité et immigration».

44. Ce trait passait d'autant plus inaperçu qu'il s'appliquait à des populations minoritaires, les plus défavorisées, les plus désaffiliées, les moins susceptibles de produire un contre discours. Depuis la crise de 2008, on voit apparaître un discours criminologique s'en prenant au $1 \%$ les plus riches avec des argumentaires qui dupliquent ceux utilisés pour la stigmatisation des étrangers en situation irrégulière, des déboutés du droit d'asile ou des populations nomades. Ils seraient tous « mobiles ", « sans attache au sol », « sans identité nationale bien construite ", etc. Cela confirmerait d'une certaine façon le diagnostic de Zygmunt Bauman sur les conséquences de la mondialisation mais entrainerait une sorte de renversement d'analyse par rapport à la situation de privilégiés qu'il décrivait auparavant. La majorité des « envieux » de la mondialisation pourraient ainsi se retourner, non seulement contre les vagabonds et les touristes pauvres, mais aussi contre les « élites ». Une certaine criminologie en France qui se situait très à droite de l'échiquier politique au milieu des années $2000 \mathrm{a} \mathrm{pu}$ ainsi rapidement réinvestir le terrain de son utilité politique, en visant le cyber-crime, le crime financier, la cor- 
La raison criminologique contemporaine apparaît plus technique, et surtout plus gestionnaire qu'elle ne l'était à ses origines. Elle est très sourcilleuse des formes que prennent ses raisonnements, tout en infléchissant très peu ses objectifs. Elle ne revient donc pas sur son caractère de "science appliquée » permettant d'étudier le phénomène criminel, mais elle s'ajuste aux modes de raisonnement du libéralisme et à ses outils de gestion et de communication pour convaincre de son utilité et de sa "vérité ». La politique des chiffres devient dès lors cruciale, tant dans le style de pensée que dans l'action. Et l'on comprend mieux les efforts des tenants de la nouvelle criminologie française pour conquérir et monopoliser les institutions de mesure de la délinquance, comme l'ONDRP par exemple ${ }^{45}$.

La gestion des populations via les outils statistiques propose une vision et des modes de production de la vérité qui se veulent plus «neutres » que la criminologie centrée sur l'individu dangereux. Ils n'essentialisent pas la frontière entre le criminel et les autres, mais essaient de l'établir en termes de proportion au sein d'une population donnée. Cela permet ainsi de s'éloigner du psycho-biologique et de renouer avec «l'économie du crime », c'est-à-dire « l'opportunité » et les calculs « coûts/avantages » au cœur des approches de microéconomistes comme Gary Becker ${ }^{46}$. Dans cette " criminologie du soi ", comme l'appelle David Garland, le délinquant apparaît comme un consommateur rationnel, un « homme situationnel 47 ». L'infléchissement discursif se fait en faveur des situations risquées, de la vulnérabilité des victimes et prend ses distances avec le profil individuel, sauf pour les cas de récidive (de réincidence ou de réitération) ${ }^{48}$. La figure du récidiviste mêle en effet cette rationalité libérale et celle de l'individu dangereux ( "la criminologie de l'autre » pour Garland) dont il faudrait explorer le psychisme et évaluer le danger potentiel, même après la peine, dans des « diagnostics à visée criminologique » par exemple ${ }^{49}$.

À cette exception près, la raison criminologique recomposée a su trouver, avec son ajustement aux normes statistiques d'abord, son investissement dans l'économie des sentiments des populations ensuite, puis dans le discours de la prévention situationnelle et sa technicisation via la cartographie du «crime »,

ruption via l'image des "banksters », se donnant par là un vernis acceptable dans une partie de la gauche critique. Voir notamment Bauer A., Crime 3.0: the rise of global crime in the 21st century, Washington, Westphalia Press, 2014.

45. Pour l'usage des chiffres au niveau des politiques locales de sécurité, voir l'article d'Élodie Lemaire et Laurence Proteau dans ce numéro.

46. Becker G., Essays in the Economics of Crime and Punishment, New York, Columbia University Press, 1974.

47. Garland D., "Les contradictions de la "société punitive". Le cas britannique ", Actes de la recherche en sciences sociales, 124, 1998, pp. 49-67.

48. Harcourt B.E., Against Prediction. Profiling, Policing, and Punishing in an Actuarial Age, Chicago/London, The University of Chicago Press, 2006.

49. Voir dans cc numéro les contributions respectives de Véronique Voruz sur la psychiatrie et d'Olivier Razac et Fabien Gouriou sur les services pénitentiaires d'insertion et de probation (SPIP). 
les appuis politiques qu'elle avait perdu dans les années 1960, lorsque son modèle de prophylaxie sociale apparaissait comme le lieu par excellence des nostalgiques du colonialisme et même de l'eugénisme et du fascisme. Barrée pour un temps des cercles politiques et universitaires au profit d'une démarche de sociologie policière et judiciaire se développant à l'abri des renouvellements du droit pénal, elle connut un retour en grâce à partir de la fin des années 1970, lorsque «l'insécurité » devint un objet et un enjeu politique. Quand la sécurité acquit le statut de « première des libertés »-comme le répèteront à l'envi nombre de gouvernants de tous bords quelques années plus tard - sa subordination aux notions de liberté et de justice (comme moyen en vue de fins la dépassant) fut remise en cause. Au-delà des statistiques du crime, un savoir basé sur des enquêtes d'opinion concernant le "sentiment d'insécurité » et des études de sécurité (intérieure) pouvaient prospérer, alors justement que la sociologie du droit pénal de l'époque refusait de les cautionner, en insistant sur l'individualisation du droit, l'impossible mise en chiffre des résultats des jugements et le danger de traiter la justice à l'aune de son efficacité, ramenée elle-même à son seul caractère coercitif d'emprisonnement et de garde à vue préventive.

Si de nombreux détracteurs de la criminologie mettaient alors l'accent sur son passé et sur la pérennité d'une discipline à vocation de stigmatisation des groupes les plus faibles (folk devils) et de construction de stéréotypes sur les individus dangereux, peu ont vu les changements structurels qui furent la condition de son succès politique quand elle se para des couleurs de l'expertise statistique sur des groupes de population en fonction de leurs trajectoires et délaissa sa chasse au corps et à l'esprit criminel individuel. Plus grave, sans doute, bien des critiques de la prophylaxie sociale partageaient avec elle l'idée de l'anormalité du crime. Caressant l'idée de vivre dans un monde qui en serait débarrassé, ils renouaient avec la volonté thérapeutique en se référant au marxisme sur les causes structurelles du crime ou à la psychanalyse sur ses causes individuelles. Ils ne faisaient donc guère ce travail historique et généalogique par lequel le chercheur retrace, comme dit Foucault, «l'histoire de la manière dont les choses font problème 50 » et dont chaque narration se veut, malgré ses différences, un accès à l'universel de l'explication. Et c'est sans doute là que le philosophe intervint, en signalant certains de ces glissements et manquements des critiques classiques. Il proposa de penser les limites de la loi dans leur rapport à la biopolitique des populations et dans la problématisation des modes de gouvernement du crime. Comme le soulignent Colin Gordon et Fabienne Brion ${ }^{51}$, Foucault a eu un rapport discontinu avec le droit pénal et public et s'est très peu intéressé au discours criminologique en tant que tel, qu'il considérait comme une vacuité. Mais, à l'invitation des juristes belges luttant contre les mesures de sûreté que le gouvernement voulait instituer, il

50. Voir son entretien dans ce numéro.

51. Voir leurs deux contributions dans ce numéro. 
prit une position politique de soutien et accepta de venir faire cours à l'Université catholique de Louvain en 1981, tout en se mettant à distance du militantisme par un propos rappelant la généalogie des modes de gouvernement par la loi et par la vérité et n'abordant que dans la toute dernière leçon la question de l'actualité ${ }^{52}$. Il visait semble-t-il à faire comprendre à son public que les arguments de souveraineté, de fondement juridique de la loi, aussi importants soient-ils, ne posaient pas la question de l'exercice du pouvoir, de son fonctionnement. Pour lui, il fallait dès lors s'intéresser aux pratiques de gouvernement s'appuyant sur la gestion différentielle des populations pour analyser comment se forment et se conforment les nouvelles images sociales des anormaux et comment elles se nouent entre elles en lissant l'arbitraire de leurs significations et en se présentant comme continuités d'une même vérité universelle du crime ${ }^{53}$. Il lui semblait donc que l'essentiel était ailleurs que dans les débats entre une criminologie prophylactique et une criminologie critique. Il s'agissait de questionner l'acceptabilité des pratiques punitives contemporaines et d'ouvrir une brèche dans le régime de légitimité et de rationalité qui les sous-tend.

Si la critique qu'il propose en suivant la piste de la généalogie de l'idée d'individus dangereux s'applique assez bien aux débats de son temps, elle n'est plus aussi pertinente pour traiter des évolutions contemporaines du discours gestionnaire et statisticien de la criminologie qui s'est éloigné de l'individu pour se recentrer sur l'analyse des situations et des profils criminels. En revanche, un point reste d'une criante actualité : la conjugaison entre le savoir statistique sur le risque et un régime de véridiction qui puise au plus profond de la pastorale chrétienne le lien entre le crime et le mal. Si la criminologie continue de fasciner et de séduire des publics divers, c'est d'une certaine façon parce qu'elle propose un horizon de libération. Le crime comme le mal pourraient être éradiqués. Un fil d'Ariane lie les différentes facettes de la raison criminologique prophylactique et ses transformations dans le temps, y compris parfois chez ceux qui s'intitulent critiques. Il est celui d'une société sans crime qui serait possible et souhaitable, si les institutions d'État le voulaient et étaient efficaces grâce à la mobilisation de savoirs, de " partenaires » locaux ou privés, de la technologie, etc. Il dessine une société de sécurité maximale à venir, une société du bonheur pour tous, une fois mis à l'écart, enfermés ou répudiés les anormaux ou les situations anormales. Cette (dé)raison s'articule alors sur un mode de véridiction faisant du crime la manifestation concrète $\mathrm{du}$ mal, et dont les visions les plus technicistes et les plus managériales ne se déprennent pas. L'utopie d'une société « guérie » du crime, omniprésente dans les discours politiques - des plus hauts responsables gouvernementaux jusqu'aux plus humbles édiles des petites villes - se décline aussi dans les

52. Foucault M., Mal faire, dire vrai: fonctions de l'aven en justice, Louvain-la-Neuve, Presses Universitaires de Louvain, 2012.

53. Cette piste est explorée pour les illégalismes financiers dans l'article d'Anthony Amicelle, dans ce numéro. 
déclarations de guerre récurrentes contre la drogue, la terreur, les bandes ou la récidive. Elle apparaît comme une profonde théologisation de la politique la plus ordinaire, qui dépolitise par son aura techniciste et prophylactique le problème de la sécurité, ses modes de raisonnement et sa légitimité.

Cette utopie a elle-même généré sa propre critique dystopique d'un monde génétiquement maîtrisé et dès lors conforme à un ordre social d'où toute innovation et déviance sont bannies, en sus du crime ${ }^{54}$. Elle a aussi produit plus récemment une mise en question de l'activité policière ordinaire. Dès lors que le mal n'est plus à rechercher dans les profondeurs de la conscience humaine (chez les usual suspects) mais dans les situations et les opportunités que des individus, aussi banals soient-ils, rencontrent, les actes futurs peuvent être anticipés, par des précogs dans The Minority Report, l'ouvrage de Philip K. Dick, mais aussi par des logiciels comme PredPol (pour Predictive Policing), utilisé par la police de Los Angeles, aux États-Unis, pour déployer ses patrouilles ${ }^{55}$. La littérature d'anticipation politique apparaît ici comme une forme de critique lucide et efficace de cette version conservatrice des lendemains qui chantent que la criminologie décline comme un avenir « où vous n'aurez plus peur et serez libéré du mal », afin de justifier au quotidien des politiques de gestion de l'inquiétude, dont l'une des forces est de se manifester aussi sous les traits de la sollicitude, de la bienveillance, et non de la coercition directe.

\section{Tensions critiques}

À rebours de cette criminologie et de ses récents développements hexagonaux coexistent ceux qui présentent la criminologie critique comme une véritable alternative et ceux qui doutent de la pertinence de toute forme de raisonnement criminologique.

Dans d'autres pays que la France, parce que la criminologie a su faire émerger des formes de savoir plus respectueux des règles de la connaissance académique et de la vérité historique, des auteurs insistent sur la validité d'une discipline autonome, dès lors qu'elle suit le projet que Foucault donne dans l'entretien qu'il avait accordé à André Berten et que nous republions dans ce numéro. «Interroger le système pénal moderne à partir de la pratique punitive, de la pratique corrective, à partir de toutes ces technologies par lesquelles on a voulu modeler, modifier, et catera, l'individu criminel, il me semble que ça permet sans doute de faire apparaître un certain nombre de choses ", explique-t-il. On peut suivre le cheminement de sa pensée, qui peut déboucher sur deux lignes d'interprétation. La première invite à prendre la mesure d'une institution à partir non pas du discours sur lequel elle se fonde, mais de

54. Des romans comme Le Meilleur des Mondes d'Aldous Huxley (1932) ou le film Bienvenue à Gattaca d'Andrew Nicoll (Colombia pictures, 1997) symbolisent ce mouvement.

55. Voir http://www.predpol.com 
ce qu'elle fait - de ses « effets ». Il faut voir qui est en prison, ce que la prison fait à ceux qui y ont été et/ou y sont et, à partir de là, se demander ce qu'est la loi. La seconde suit la problématique suivante : quelles sont les conduites qui influent sur les conduites dites légales ? Comment les historiciser, en faire la généalogie afin de les fragiliser dans leurs certitudes et les questionner ? La criminologie critique serait alors cette œuvre de fragilisation des certitudes du droit pénal et des pratiques punitives qu’opère le travail historique généalogique. Paradoxalement, elle serait alors une " anti-criminologie », car la connaissance qu'elle produit devient insupportable pour une discipline qui, dans sa version prophylactique, se veut préventive et prédictive. La généalogie replace l'ambiguïté au cœur de la pratique et déstabilise les croyances converties en certitudes - sur lesquelles reposait l'édifice. Il n'y aurait donc pas de criminologie critique foucaldienne. Il ne pourrait y avoir qu'une approche foucaldienne qui se situe hors des limites de la criminologie disciplinaire et en moque les frontières mouvantes qui se cristallisent à chaque fois comme de nouvelles barrières de protection pour ceux qui désignent ce qu'est le crime (criminel né, criminel comportemental, action criminelle, opportunité d'action dans une situation donnée, etc.), à l'égard des procédures de véridiction.

Pour des sociologues de la déviance, en revanche, le doute sur toute forme de savoir criminologique prend une tournure plus radicale. Howard S. Becker le résume abruptement : "Les criminologues n’ont jamais rien fait à propos du problème du crime ». Un criminologue, même critique, s'implique dans le système de justice criminelle et y participe, y compris en le réformant. Or, y participer, c'est concourir à son hypocrisie. L'étude du système de justice pénale et de ses différentes composantes (police, justice, prison) ne peut alors relever que d'une sociologie plus générale des institutions. Une sociologie qui vise à penser réflexivement la construction des catégories de ces institutions, quand elles parlent du crime, et qui analyse la réalité de leurs pratiques et de leur tendance à vouloir perdurer selon les intérêts professionnels de leurs agents. Une sociologie attentive aux manières dont elles construisent les statistiques qui ensuite leur servent de base pour proposer les raffinements à apporter à leurs savoirs en constitution, qu'ils portent sur les comportements humains via les neurosciences ou sur la gestion des populations potentiellement criminelles via des statistiques et des algorithmes, toujours présentés comme suffisamment bons (good enough) à l'égard de la « réalité du crime » présent et à venir, mais toujours aussi perfectibles, afin de maintenir la pression sur "l'innovation ». Ici, la critique rejoint une sociologie des formes contemporaines des savoirs d'État, dont les progrès technologiques (et notamment numériques) ont exacerbé l'ambition prédictive.

Les contributions rassemblées dans ce numéro mettent en tension ces deux lignes d'analyse en les déployant sur des terrains spécifiques et en les articulant chacune à leur manière. Ensemble, elles dessinent la ligne de pente 
d'une raison criminologique qui, malgré ses ajustements, demeure relativement stable dans le temps. Dès lors que l'on accepte l'analyse de Durkheim du crime comme normal et nécessaire, l'obsession prophylactique de celle-ci semble vouée à " poursuivre désespérément une fin qui fuit à mesure qu'on avance $56 »$. En revanche, si l'on prend au sérieux le caractère ontologique qu'elle donne au crime, le travail de classification des groupes et des individus qu'elle opère en fonction non pas seulement de leurs actes, mais de leur possibilité de les commettre, les mesures qu'elle promeut en deçà et au-delà du droit pénal afin d'anticiper, de prévenir et de « défendre la société », tout comme la définition et la hiérarchisation permanente des menaces auxquelles elle se livre, la raison criminologique apparait pour ce qu'elle est : une rationalité de gouvernement profondément conservatrice, toute entière tendue vers la préservation de l'ordre des choses, c'est-à-dire de l'ordre social et politique tel qu'il est. 